\title{
Aprendizaje cooperativo a través de las nuevas tecnologías: Una revisión
}

\author{
Cooperative learning through new technologies: A review
}

\section{Rosario Isabel Herrada Valverde}

Universidad de Almería

rherrada@ual.es

\section{Raúl Baños Navarro}

Universidad de Almería

rbanos@ual.es

Fecha presentación: 21/12/2017 | Aceptación: 13/04/2018 |Publicación: 22/06/2018

\begin{abstract}
Resumen
Las Tecnologías de la Información y la Comunicación han supuesto una auténtica revolución en el ámbito educativo, provocando cambios estructurales en la forma de enseñar y en la manera de aprender. Actualmente, la existencia de una gran variedad de plataformas virtuales de enseñanza-aprendizaje permiten al profesorado y alumnado interactuar de forma síncrona o asíncrona haciendo uso de ordenadores y dispositivos móviles. Esto ha favorecido la implementación de metodologías activas de enseñanza-aprendizaje de contrastada eficacia pedagógica que, como es el caso del aprendizaje cooperativo, favorecen que los estudiantes adquieran competencias y mejoren sus resultados académicos. Pese al interés del tema, no existen revisiones actualizadas sobre experiencias didácticas e investigaciones en las que se promueva el aprendizaje cooperativo haciendo uso de las nuevas tecnologías y que hayan sido redactadas en idioma español. Con el objetivo de dar respuesta a esta demanda, se revisan un gran número de estudios y experiencias didácticas existentes en la bibliografía en diferentes materias y niveles educativos. A partir de dicha revisión se puede concluir que existe un creciente interés por parte de docentes e investigadores a la hora de implementar el aprendizaje cooperativo haciendo uso de plataformas virtuales de enseñanza-aprendizaje, así como de redes sociales o cursos MOOC, a la vez que se observa un elevado nivel de satisfacción por parte de docentes y discentes involucrados en dichas experiencias.
\end{abstract}

Palabras clave: tecnología educativa; innovación educativa; educación a distancia; actividades grupales; aprendizaje cooperativo

\begin{abstract}
Resum
Les Tecnologies de la Informació i la Comunicació han suposat una autèntica revolució en l'àmbit educatiu, provocant canvis estructurals en la forma d'ensenyar i en la manera d'aprendre. Actualment, l'existència d'una gran varietat de plataformes virtuals d'ensenyament-aprenentatge permeten al professorat i alumnat interactuar de forma síncrona o asíncrona fent ús d'ordinadors i dispositius mòbils. Això ha afavorit la implementació de metodologies actives d'ensenyament-aprenentatge de contrastada eficàcia pedagògica que, com és el cas de l'aprenentatge cooperatiu, afavoreixen que els estudiants adquireixin competències i millorin els seus resultats acadèmics. Malgrat l'interès del tema, no existeixen revisions actualitzades sobre experiències didàctiques $i$ investigacions en què es promogui l'aprenentatge cooperatiu fent ús de les noves tecnologies i que hagin estat redactades en idioma espanyol. Amb l'objectiu de donar resposta a aquesta demanda, es revisen un gran nombre d'estudis i experiències didàctiques existents a la bibliografia en diferents matèries i nivells educatius. A partir d'aquesta revisió es pot concloure que hi ha un creixent interès per part de docents i investigadors a l'hora d'implementar l'aprenentatge cooperatiu fent ús de plataformes virtuals d'ensenyament-aprenentatge, així com de xarxes socials o cursos MOOC, alhora que s'observa un elevat nivell de satisfacció per part de docents i discents involucrats en aquestes experiències.
\end{abstract}

Paraules clau: tecnologia educativa; innovació educativa; educació a distància; activitats grupals; aprenentatge cooperatiu 
Herrada Valverde, Rosario Isabel y Baños Navarro, Raúl (2018). Aprendizaje cooperativo a través de las nuevas tecnologías: Una revisión. @tic revista d'innovació educativa, 20, 16-25.

\begin{abstract}
Information Communication Technologies have brought a revolution in education, causing structural changes in the way we teach and how we learn. Currently, the existence of a wide variety of virtual teaching-learning environments enables teachers and students to interact synchronously or asynchronously using computers and mobile devices. This has led to the implementation of active teaching-learning methodologies with proven pedagogical effectiveness, such as cooperative learning, that encourage students to acquire skills and improve their academic performance. Despite the importance of the subject, almost no papers have analysed the state-of-the-art on educational experiences that promote cooperative learning using new technologies. In order to respond to this demand, this paper presents a comprehensive review of the related bibliography that include a large number of papers written in Spanish in which new technologies are used to implement cooperative learning activities in different subjects and educational levels. From the analysis of these papers, it can be concluded that there is a growing interest by teachers and researchers in implementing cooperative learning using virtual teaching-learning environments and computer applications, as well as social networks or MOOC. Further, these experiences receive positive feedback from teachers and students involved.
\end{abstract}

Key Words: educational technology; educational innovation; distance education; group activities; cooperative learning

\section{Introducción y estado de la cuestión}

Durante las últimas décadas, venimos asistiendo a una continua renovación metodológica que pretende orientar el proceso educativo hacia lo que podríamos llamar un "enfoque centrado en el alumno" (Silva, 2017), según el cual los alumnos dejan de ser meros receptores de la información que les transmite el docente para pasar a jugar un papel central en su propio proceso de aprendizaje (Danker, 2015). Para ello, se suele recurrir a las metodologías activas de enseñanza-aprendizaje, entre las que encontramos el aprendizaje cooperativo (Johnson, Johnson y Holubec, 1999), el estudio de casos (Aramendi, Bujan, Garín y Vega, 2014), el aprendizaje basado en problemas (Santillán-Campos, 2013), el aprendizaje basado en proyectos (García-Valcárcel y Basilotta, 2017), la clase invertida (Sánchez-Vera, Solano y Calatayud, 2016), o esquemas que combinan diferentes metodologías (González y Carrillo, 2016).

Centrándonos en el aprendizaje cooperativo (AC), esta metodología se basa en el empleo didáctico de grupos reducidos en los que los alumnos trabajan juntos para alcanzar objetivos comunes, maximizando su propio aprendizaje y el de los demás miembros del grupo (Johnson, Johnson y Holubec, 1999; Pujolàs, 2008), frente al aprendizaje individualista y competitivo intrínseco a la metodología tradicional. Existen diferentes modelos de referencia a la hora de diseñar tareas cooperativas (Mondéjar, Vargas y Meseguer, 2007), incluyendo la técnica del puzzle o rompecabezas (Jigsaw), el aprendizaje por equipos de estudiantes (Student team learning), la técnica de aprender juntos (Learning together), y la investigación en grupo (Group investigation). La metodología de AC debe incorporar cinco elementos básicos: interdependencia positiva, responsabilidad individual, interacción cara a cara, habilidades interpersonales y supervisión grupal (Del Pozo y Horch, 2008). El AC fomenta las relaciones interpersonales, y ayuda a desarrollar habilidades de búsqueda, selección, análisis y evaluación de la información, mejorando la percepción de la realidad (Llopis, 2011). Algunos especialistas consideran que el AC debería ser un contenido escolar más que los alumnos tendrían que aprender a lo largo de su escolaridad (Pujolàs, 2008). Esta metodología ejemplifica la renovación pedagógica propia de sociedades plurales y participativas (Gozálvez, García y Traver, 2011), ya que fomenta una mayor participación, comunicación, implicación y responsabilidad por parte de los alumnos, mejorando la convivencia, desarrollando habilidades sociales y valores entre el alumnado, y favoreciendo la mejora de los resultados académicos (Borràs, Sabater y Socías, 2017).

La introducción de las Tecnologías de la Información y la Comunicación (TIC) en la educación favorece el desarrollo de nuevas teorías y prácticas didácticas, modificado los roles de profesores y alumnos, hasta el punto de que algunos autores hablan de "pedagogías emergentes" (Adell y Castañeda, 2015). Así, algunos estudios han demostrado que la TIC están provocando innovaciones en la práctica docente de una parte importante del profesorado, incluyendo el desarrollo de nuevos métodos y estrategias de enseñanza, entre las que se encuentra el AC (Bartau, Azpillaga y Joaristi, 2017). Las TIC son recursos didácticos, instrumentos para la organización, gestión y administración educativa que estimulan la comunicación, la mediación y la construcción compartida del conocimiento (Salmerón, Rodríguez y Gutiérrez, 2010). De hecho, las TIC ayudan a superar las barreras espacio-temporales tradicionales, ya que las plataformas virtuales de enseñanza-aprendizaje (Maraza, 2016) permiten al profesorado y alumnado interactuar de forma asíncrona, sin necesidad de coincidir física y temporalmente en el aula. Ello ha permitido el desarrollo de modelos de enseñanza no presencial (e-learning), así como semipresencial (blended learning, b-learning), en los que se combinan procesos de enseñanza-aprendizaje cara a cara con tareas y actividades a distancia (González, Perdomo y Smith, 2017). Para que las TIC sean eficaces en el ámbito educativo, es necesario que las instituciones formativas promuevan su uso, favoreciendo una educación virtual humana e inclusiva (Leiva y Almenta, 2013); que los docentes posean destrezas en su manejo (Sáez, 2010; Palomares y Chisvert, 2016); y que los alumnos se organicen adecuadamente construyendo un sentimiento de comunidad (Tirado, Aguaded y Méndez, 2009), mostrando una actitud crítica y reflexiva a la hora de seleccionar y utilizar las herramientas tecnológicas y las fuentes de información. Según García-Valcárcel y Tejedor (2017), las diferencias en el rendimiento de los alumnos depende en gran medida de la valoración que hacen de las TIC para mejorar la elaboración de trabajos, de la organización de su actividad académica, de las tareas de repaso, así como de la búsqueda de recursos. Por todo lo anterior, y teniendo en cuenta que las TIC son de gran utilidad para la implementación de metodologías activas (González y Carrillo, 2016), procedemos a analizar el uso de las nuevas tecnologías para promover el aprendizaje cooperativo. 
Herrada Valverde, Rosario Isabel y Baños Navarro, Raúl (2018). Aprendizaje cooperativo a través de las nuevas tecnologías: Una revisión. @tic revista d'innovació educativa, 20, 16-25.

\section{Material y métodos}

La metodología utilizada en el presente estudio se basa en un modelo habitualmente utilizado en la elaboración de revisiones bibliográficas (Gómez-Luna, FernandoNavas, Aponte-Mayor y Betancourt-Buitrago, 2014), que consta de cuatro fases: definición del problema; búsqueda de la información; organización de la información; y análisis de la información. Así, el problema que abordamos es llevar a cabo una revisión bibliográfica sobre trabajos de investigación recientes que hayan abordado el estudio del aprendizaje cooperativo a través de las nuevas tecnologías, al objeto de extender estudios previos en este ámbito (Becerril, 2010; Rodríguez, Cebollada, Hueto y Molledo, 2012).

En cuanto a la búsqueda de información necesaria para llevar a cabo el estudio, se ha hecho uso de Dialnet, que es un portal bibliográfico que incluye seis millones de documentos, y unos dos millones de usuarios registrados (Mateo, 2015). En concreto, se ha realizado una búsqueda de artículos publicados en revistas iberoamericanas durante la última década que incluyan los términos "aprendizaje cooperativo" / "AC" $\mathrm{y}$ "tecnologías de la información y la comunicación" / "TIC" / "TICs" en el título, en el resumen, o en las palabras clave. Como resultado de esta búsqueda se han recopilado 87 artículos que cumplen estos criterios, la práctica totalidad de los cuales son incluidos en nuestro estudio. La elección prioritaria de artículos publicados en revistas viene justificada por el hecho de que se trata de documentos actualizados, especializados y revisados por expertos en la materia. Aunque, por limitaciones de espacio, no analizamos la bibliografía internacional publicada en idioma inglés, consideramos que los datos aquí analizados son suficientemente representativos para denotar la actividad docente e investigadora en este ámbito. En lo que respecta a la fase de organización de la información, indicar que los documentos recopilados han sido agrupados siguiendo un criterio temático, de forma que, por un lado, se han analizado los beneficios de las TIC a la hora de implementar el aprendizaje cooperativo, $y$, por otro, diferentes experiencias llevadas a cabo con anterioridad en las que se hace uso de herramientas TIC, incluyendo el uso de plataformas virtuales de enseñanza-aprendizaje, herramientas de gestión de contenidos, herramientas de comunicación, así como el uso de redes sociales y MOOC. Por último, la fase de análisis de la información ya organizada se ha centrado en identificar las aportaciones de las publicaciones recopiladas.

\section{Análisis y resultados}

A continuación, se analizan las principales aportaciones de las publicaciones recopiladas. En primer lugar, se analizan las posibilidades pedagógicas que ofrecen las TIC para mejorar la eficiencia del AC. Posteriormente se describen las diferentes plataformas virtuales de enseñanza-aprendizaje para la implementación del AC en el ámbito educativo. También se describen diferentes herramientas de gestión de contenidos y herramientas de comunicación que han sido utilizadas en este contexto. Por último, se hace mención a las posibilidades que ofrecen las últimas tendencias de interacción socioeducativa, como son las redes sociales y los MOOC, y su uso para llevar a cabo experiencias basadas en AC.

\subsection{Utilidad pedagógica de las TIC en el aprendizaje cooperativo}

Los entornos virtuales de enseñanza-aprendizaje son espacios formados por herramientas y recursos que permiten a los sujetos intervenir e interactuar mediante comunicación uno a uno, uno a muchos o muchos a muchos (Nuñez, 2011), siendo las características formales de intercambio de información diferentes a la interacción cara a cara. Según Pulido, Martín-Seoane y Lucas-Molina (2013), las tecnologías virtuales permiten una representación temporal y espacial que permite a los alumnos reconfigurar sus oportunidades y estrategias de acción al aprender en equipo. Las TIC potencian competencias interpersonales, instrumentales, y sistémicas, razón por la cual la existencia de comportamientos estratégicos de los estudiantes durante el desarrollo de tareas cooperativas (López y Álvarez, 2011) hace que el uso combinado de las TIC y del AC mejore el rendimiento de los grupos tradicionales (Méndez, 2015), en línea con experiencias llevadas a cabo a nivel preuniversitario (Jiménez, Llitjós y Puigcerver, 2007) y universitario (Leris y Sein-Echaluce, 2009), tanto en materias de humanidades y ciencias sociales (Cano et al., 2010), como de ciencias e ingeniería (Niculcar, Fernández, Gracia, García y González Benítez, 2009). Guitert y Pérez-Mateo (2013) describen los factores de carácter teórico que influyen sobre el aprendizaje colaborativo en entornos virtuales. Diferentes autores ofrecen orientaciones sobre la implementación del AC en redes y entornos virtuales de comunicación asíncrona (Casanova, Álvarez y Gómez, 2009; Maraver, Hernando y Aguaded, 2012). Otros aspectos clave son la formación, coordinación y cooperación entre docentes, o la disponibilidad de recursos materiales adecuados (Sáez, 2010). Algunas investigaciones remarcan las posibilidades que ofrece el AC apoyado por las TIC para dar respuesta a la interculturalidad en las aulas (Pantoja y Blanco, 2011), como, por ejemplo, ayudar a superar dificultades que algunos estudiantes tienen en asignaturas de lengua (Ortega, 2015). Las TIC favorecen el uso de las rúbricas electrónicas (e-rúbricas) en la evaluación del aprendizaje cuando se aplica el AC mediante tareas y proyectos (Cebrián, Serrano y Ruiz, 2014). La sinergia entre las estrategias cooperativas y las TIC también resultan de utilidad más allá del aula, al facilitar la creación de espacios de cooperación entre instituciones (Rodríguez y De Miguel, 2007). Todo lo anterior se ve respaldado por la opinión positiva mostrada por los estudiantes acerca del uso del AC con soporte de las TIC (Herrero et al., 2013).

\subsection{Plataformas virtuales de enseñanza-aprendizaje}

Los docentes comenzaron a aplicar AC asistido por ordenador al paraguas de entornos virtuales de enseñanza-aprendizaje tales como Moodle, Blackboard o WebCT, que permiten gestionar asignaturas, mediante la inclusión de contenidos multimedia y herramientas de evaluación y comunicación (Ros, 2008), siendo plataformas básicas para el desarrollo del AC y de la investigación social (González, 2011).

WebCT y Blackboard son dos de las plataformas virtuales más utilizadas. Así, León, Camarillo, Ramos y Sánchez (2008) analizaron WebCT como infraestructura tecnológica para facilitar la enseñanza virtual, considerando aspectos como la seguridad, privacidad, autenticación de usuarios. Hernando, Aguaded y Tirado (2011) destacan el papel dinamizador del profesor-tutor 
al desarrollar actividades cooperativas mediante WebCT, destacando la importancia que tiene la frecuencia de las interacciones dentro de cada grupo. Tirado, Hernando y Aguaded (2011) analizan el intercambio de mensajes a través de WebCT entre alumnos de Educación Social, concluyendo que el tamaño del grupo tiene cierta influencia en la densidad e intensidad de la comunicación. Suárez (2010) analiza la cooperación textual asíncrona que se produce en foros de discusión de Blackboard, mientras que García y Suárez (2011) hacen uso de dichos foros para llevar a cabo una investigación etnográfica virtual sobre la interacción cooperativa entre alumnos de Magisterio de Educación Infantil bajo condiciones tecnológicas de mediación.

Otra de las principales plataformas virtuales de enseñanza es Moodle, que incluye recursos para la gestión de contenidos, comunicación, y evaluación (Ros, 2008). Experiencias como la de Margelí y Muria (2008) tratan de aprovechar las cualidades de Moodle para gestionar los contenidos de una forma organizada, a la vez que permite crear comunidades virtuales de AC. Andreu, Sanz y Serrat (2009) experimentan la modalidad cooperativa conocida como grupos de investigación, en la que los alumnos se comunican con el profesor a través de Moodle para subir y descargar enunciados y soluciones de actividades. Moodle ha sido utilizado en materias de Ingeniería (Leris y Sein-Echaluce, 2009), Administración y Dirección de Empresas (Cano et al., 2010), Comunicación (Chamizo y Fernández, 2013), o Geografía (Boix y Armisen, 2008).

\subsection{Herramientas para la gestión de contenidos}

Además de las utilidades incluidas en las plataformas que acabamos de comentar, algunos docentes proponen la elaboración de libros o bibliotecas digitales a la hora de gestionar los contenidos de la materia. Es el caso de López y García (2011), quienes proponen el uso de libros virtuales como recursos educativos para desarrollar la escuela inclusiva mediante el trabajo cooperativo. García (2009) plantea a los alumnos la creación de bibliotecas digitales de forma cooperativa. Miguel (2010) implementa estrategias de AC y resolución de problemas mediante el desarrollo de glosarios de términos, promoviendo entre los alumnos la adquisición de competencias de búsqueda y comunicación telemática, evaluación crítica, y conocimiento del vocabulario. De Lucas, Martín, Haza, Delgado y Lucas (2009) usan RefWorks para que estudiantes y profesores agrupados en una red cooperativa intercambien referencias de publicaciones científicas, favoreciendo la iniciación de los estudiantes en el ámbito investigador.

Del Moral y Villalustre (2007) promueven estrategias de enseñanza basadas en el trabajo colaborativo de investigación a través de WebQuest, que es un recurso que integra las TIC con los contenidos de la asignatura, y facilita el trabajo cooperativo, autónomo y significativo. Ha sido utilizado tanto en etapas preuniversitarias (Lara y Repáraz, 2007) como universitarias (Gallego y Guerra, 2007; Osicka, Fernández, Valenzuela, Buchhamer y Giménez, 2013). Así, Hernández (2007) analiza el uso de las WebQuest por parte de profesores y alumnos para la Enseñanza de Lenguas Extranjeras, mientras que Lozano (2011) presenta una investigación en el marco de un trabajo fin de máster que analiza el desarrollo de la competencia matemática en contextos de Ciencias Sociales utilizando metodologías activas y WebQuest. Pegalajar (2009) reflexiona sobre las opciones que las TIC y el AC ofrecen para favorecer el aprendizaje de alumnos con discapacidades intelectuales, incluyendo el uso del WebQuest. Lara y Repáraz (2007) fomentan el trabajo cooperativo autónomo de los alumnos mediante la realización de vídeos científicos en Geografía a nivel de Educación Secundaria utilizando WebQuest. Gómez, Palomares y Pino (2010) presentan una experiencia en la que los alumnos de Publicidad y Relaciones Públicas desarrollan una WebQuest, observándose que la mayoría de ellos se apoyan en herramientas tales como Google Blogger, Wikispaces, Youtube o Google Maps. Siliberti (2011) analiza las posibilidades de las WebQuest, blogs, o wikis para implementar el AC en la enseñanza de lenguas extranjeras. Hernando, Aguaded y Tirado (2011) utilizan una plataforma digital basada en WebCT sobre la que los alumnos construyen una WebQuest. Guerra, Riaño y González (2008) utilizan las WebQuest y el portafolio digital con estudiantes de Educación Primaria. Moreno-Crespo, López y Cruz (2014) utilizan el formato de blog como soporte del portafolio digital para implementar el AC en una asignatura universitaria de Educación Social, lo que permite un intercambio rápido y fluido de información entre los alumnos. Otras herramientas como Courselab (Serrano, Muñoz y López, 2012) o BSCW/BSCL (Gutiérrez, Yuste, Cubo y Lucero, 2011) también han sido utilizadas para implementar el AC en el aula. Burgueño (2016) aplicó la técnica del aula invertida, de forma que los alumnos debían visualizar y analizar diferentes videos, los cuales eran accesibles a través de Google Blogger.

Diversos estudios han hecho uso de dispositivos móviles para la adquisición de contenidos. Así, por ejemplo, Jiménez (2016) presenta un proyecto llevado a cabo con alumnos de bachillerato, en el que se aplica aprendizaje cooperativo, además de otras metodologías como aprendizaje basado en proyectos, y donde las TIC juegan un papel fundamental para promover la competencia digital entre alumnos a través del uso de dispositivos móviles y la elaboración de códigos QR sobre puntos de interés. En otro estudio reciente, Monedero, Castro y Luengo (2017) Ilevaron a cabo una serie de actividades en grados de Periodismo, de Publicidad y Relaciones Públicas y en Comunicación Audiovisual en las que los alumnos, de forma cooperativa, elaboraban videotutoriales a través de Youtube a los cuales se les asociaron códigos QR ubicados en equipos y en las aulas.

\subsection{Herramientas de comunicación}

Diferentes estudios han analizado el uso de herramientas TIC para favorecer las competencias de los alumnos en tratamiento de la información y competencias digitales (Corral y Cacheiro, 2016). Los blogs y foros de Internet permiten a los estudiantes leer comentarios de otros compañeros y docentes, lo que resulta enriquecedor para la construcción de conocimiento personal (Jiménez, Llitjós y Puigcerver, 2007). García (2008) propone los weblogs como herramientas para la adquisición de competencias y el desarrollo de la identidad personal de los alumnos de Lenguas Extranjeras. Gómez (2014) presenta los resultados de un proyecto con aplicaciones tecnológicas, en el que la comunicación se lleva a cabo a través de correo electrónico, de blogs educativos desarrollados a través de Google Blogger, de Whatsapp, o de Dropbox, y donde se crearon mapas conceptuales mediante Visual Understanding Environment (VUE). Molina, Valencia-Peris y Gómez-Gonzalvo (2016) analizaron las opiniones de un gran número de estudiantes universitarios sobre el uso 
Herrada Valverde, Rosario Isabel y Baños Navarro, Raúl (2018). Aprendizaje cooperativo a través de las nuevas tecnologías: Una revisión. @tic revista d'innovació educativa, 20, 16-25.

de blogs en diferentes asignaturas, comprobando que los estudiantes consideran que dichos edublogs contribuyen positivamente al aprendizaje cooperativo.

Otra herramienta ampliamente utilizada en el ámbito educativo es el chat. Diversos autores han constatado diferencias en las características formales del intercambio de información derivadas de la diferente naturaleza del canal de comunicación utilizado, así como del tamaño de los grupos organizados (Roselli, Bruno y Evangelista, 2004). Trigueros, Rivera y De la Torre (2011), implementaron seminarios virtuales con alumnos de Magisterio de Educación Física que, haciendo uso de chats, permitan establecer espacios para el AC, favoreciendo la reflexión y discusión. Aguirre, Casco y Laurencio (2009) destacan las ventajas del chat como herramienta de innovación educativa para la construcción y gestión del conocimiento, permitiendo dar forma a grupos cooperativos.

Además de los blogs y chats, los espacios wikis también facilitan el desarrollo de actividades cooperativas. Gutiérrez, Yuste, Cubo y Lucero (2011) presentan una experiencia en la que los alumnos crean un wiki-site trabajando de forma cooperativa, de forma similar a otras implementaciones en las que se hace uso de las utilidades de Moodle (Mondéjar, Vargas y Meseguer, 2007). Espinosa (2014) analiza como la creación de wiki de forma cooperativa permite al docente observar, de forma individualizada, el proceso de escritura de los alumnos de español como lengua extranjera. Ruda (2013) plantea el uso de cuestionarios a través de Google Drive a estudiantes universitarios para analizar las motivaciones y dificultades a la hora de realizar una investigación de grupo empleando foros de Moodle y espacios de discusión de Wikispaces.

\subsection{Redes sociales}

Algunos autores han analizado el uso de redes sociales como herramientas de comunicación cooperativa con retroalimentación en tiempo real. Así, Trujillo, Cáceres, Hinojo, y Aznar (2011) recopilan opiniones positivas por parte de alumnos que utilizan una red social colaborativa interuniversitaria creada a través de una herramienta web 2.0 (Ning). Peña, Pérez y Rondón (2010) analizan las posibilidades de las redes sociales para fomentar el AC y las relaciones sociales entre los alumnos, ofreciendo un sustento teórico que evidencia principios coincidentes entre las relaciones sociales y el uso de metodologías activas. González, García y Ramírez (2015) analizan las opiniones de los alumnos sobre las posibilidades de herramientas como Google Docs, Google Drive, y las redes sociales Google+ y Twitter, para implementar el AC y la tutoría entre iguales, observando que estas ayudan a desarrollar competencias y a superar barreras físico-temporales, en especial aquellas que permiten un contacto visual (Google+). Chamizo y Fernández (2013) presentan diversas experiencias llevadas a cabo en asignaturas de grado y postgrado relacionadas con la Comunicación, que incluyen la creación por parte de los alumnos de un diccionario de conceptos a través de Moodle, así como de una actividad basada en un juego de rol en la que los alumnos se comunican a través de Twitter. Zanfrillo y Díaz (2015) promueven el uso de plataformas tecnológicas entre alumnos de Pedagogía de una universidad española y alumnos de la Licenciatura de Administración de una universidad argentina, observando la buena acogida que tuvo el uso de Google Docs como herramienta de edición compartida de documentos, y de Facebook como red social para la comunicación, al ayudar a superar las diferencias culturales y horarias existentes entre países.

\subsection{Cursos Online Masivos y Abiertos (MOOC)}

La formación a distancia a través de los Cursos Online Masivos y Abiertos (MOOC) ha cobrado una gran relevancia en los últimos años (Cabero-Almenara, MarínDíaz y Sampedro-Requena, 2017), ya que permiten seguimientos masivos de una misma actividad formativa aprovechando las posibilidades de los sistemas digitales (García-Aretio, 2015).

Algunas investigaciones analizan el uso del AC bajo este formato. Así, Sánchez (2014) plantea la implementación de la técnica del puzzle, además de utilizar otras herramientas como foros, video, códigos QR, elementos de hipertexto y multimedia, elaborados a través de Moodle, en el diseño de un curso MOOC. Castaño, Maiz y Garay (2015) muestran la valoración positiva de los participantes en un $\mathrm{MOOC}$ de carácter cooperativo en el que se utilizan redes sociales y herramientas como Twitter, Facebook, Linkedin, Youtube, Skype, foros, y blogs. En esta línea, Castaño, Maiz y Garay (2015) sugieren que los MOOC tienen mayor aceptación en entornos de aprendizaje ligados a la formación continua y al desarrollo profesional. Sarabia (2016) concluye que la aceptación y resultados de los estudiantes en un MOOC mejora gracias al uso del aprendizaje cooperativo. Cabe señalar, así mismo, que algunos estudios recientes reflexionan sobre la necesidad de establecer mecanismos para la evaluación del elevado número de personas que suelen atender a los MOOC (Sánchez-Vera, González y Prendes, 2017).

Al objeto de facilitar la búsqueda de documentos sobre aspectos temáticos específicos, la tabla 1 presenta un resumen de las aportaciones comentadas anteriormente, siguiendo una clasificación basada en los apartados previos.

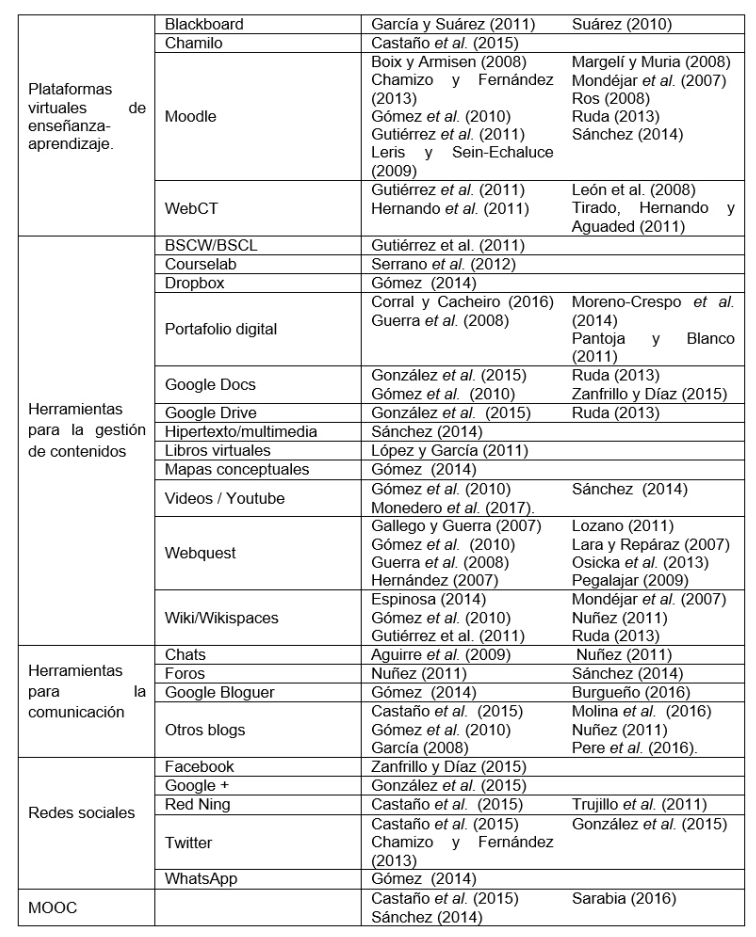

Tabla 1. Plataformas y herramientas TIC utilizadas en el aprendizaje cooperativo. Fuente: Elaboración propia 
Herrada Valverde, Rosario Isabel y Baños Navarro, Raúl (2018). Aprendizaje cooperativo a través de las nuevas tecnologías: Una revisión. @tic revista d'innovació educativa, 20, 16-25.

\section{Conclusiones}

El uso generalizado de las TIC ha facilitado la creación y difusión de materiales docentes, así como cambios en los procesos educativos. En la bibliografía encontramos numerosas investigaciones sobre metodologías didácticas de enseñanza-aprendizaje, así como estudios que analizan el papel de las TIC en la educación, aunque no encontramos revisiones recientes que analicen esta temática en idioma español, que es la lengua hablada por más de cuatrocientos millones de habitantes. Para dar respuesta a esta demanda, se revisa un amplio número de investigaciones publicadas en la última década, al objeto de ofrecer una guía comprensible para aquellos docentes e investigadores interesados en aprovechar las oportunidades que ofrecen las TIC a la hora de implementar el AC u otras metodologías activas en diferentes materias y niveles educativos.

Del análisis de las experiencias recopiladas se han obtenido una serie de conclusiones. La primera es que el uso de las TIC en educación favorece la aplicación de estrategias de enseñanza-aprendizaje alternativas a la metodología tradicional basada en la clase expositiva magistral, ya que se eliminan las barreras espaciotemporales al permitir la interacción entre docentes y discentes sin necesidad de reunirse en el mismo espacio físico de forma simultánea. Otra conclusión importante es que la implementación del AC haciendo uso de las TIC favorece el uso responsable de estas últimas, ya que se fomenta una participación activa y respetuosa entre docentes y discentes. De las experiencias analizadas también se desprende que la sinergia entre AC y las TIC mejora significativamente el clima de trabajo en el aula, promueve de forma efectiva la adquisición competencias, incrementa el interés del alumnado sobre las materias objeto de estudio, y mejoran los resultados académicos. Por otro lado, dichas investigaciones también ofrecen sugerencias, tales como la necesidad de mejorar la formación de los docentes a la hora de aplicar de forma efectiva el AC en entornos virtuales, así como la necesidad de establecer procedimientos de evaluación que tengan en cuenta las características propias del AC y de las herramientas TIC utilizadas, no sólo de las plataformas e-learning, sino también de las redes sociales o de los MOOC.

Por último, cabe señalar que la principal limitación del estudio viene dada por el hecho de que la bibliografía analizada se ciñe a publicaciones redactadas en castellano. No obstante, se han recopilado y analizado un elevado número de trabajos al objeto de que dicha limitación no sea óbice para que se evidencien las posibilidades derivadas del uso del aprendizaje cooperativo mediado por las TIC."

\section{Bibliografía}

Adell, J. y Castañeda, L. (2015). Las pedagogías escolares emergentes. Cuadernos de Pedagogía, 462, 21-25. Recuperado de: https://goo.gl/3LVY9P

Aguirre, P.C., Casco, J. y Laurencio, R.L. (2009). El Internet Relay Chat (IRC) como herramienta estratégica de innovación en la enseñanza abierta. El caso de la licenciatura en Comunicación, Universidad Veracruzana. Zona Próxima: Revista del Instituto de Estudios Superiores en Educación, 10, 50-69. Recuperado de: http://goo.gl/6ds5vd.

Andreu, L., Sanz, M. y Serrat, E. (2009). Una propuesta de renovación metodológica en el marco del Espacio
Europeo de Enseñanza Superior: los pequeños grupos de investigación cooperativos. Revista Electrónica Interuniversitaria de Formación del Profesorado, 12(3), 111-126. Recuperado de: http://goo.gl/H6mGWE

Aramendi, P., Bujan, K., Garín, S.M. y Vega, A. (2014). Estudio de caso y aprendizaje cooperativo en la universidad. Profesorado: Revista de Curriculum y Formación del Profesorado, 18(1), 413-429. Recuperado de: https://goo.gl/yZ79hU

Bartau, I., Azpillaga, B. y Joaristi, L.M. (2017). Metodología de enseñanza en centros eficaces de la Comunidad Autónoma del País Vasco. Revista de Investigación Educativa, 35(1), pp. 93-112. DOI: http://dx.doi.org/10.6018/rie.35.1.225141

Becerril, L. (2010). Orientaciones educativas sobre el uso de las TIC como apoyo al aprendizaje cooperativo en la educación escolar. Comunicación y Pedagogía: Nuevas Tecnologías y Recursos Didácticos, 241, 1921.

Boix, G. y Armisen, S. (2008). Nuevo enfoque de la práctica educativa del Programa UNIGIS Girona. Algunas reflexiones sobre las claves del cambio. Educar, 41, 41-50. Recuperado de: http://ir.uv.es/IXBtOip

Borràs, M.P., Sabater, M.V. y Socías, M.M. (2017). Cómo puede el aprendizaje cooperativo modificar la organización en la escuela. Aula de innovación educativa, 260, 55-59. Recuperado de: https://goo.gl/HBS2RM

Burgueño, J. (2010). Una chispa pedagógica. Padres y Maestros, 388, 47-52. DOI: pym.i368.y2016.008

Cabero-Almenara, J., Marín-Díaz, V. y SampedroRequena, B.E. (2017). Aportaciones desde la investigación para la utilización educativa de los MOOC. Revista Española de Pedagogía, 75(266), 727. DOI: https://doi.org/10.22550/REP75-1-201701

Cano, E.I., Chamizo, J., García, J., Jiménez, P., Martín, T., Bonilla, M.I., Martín, C. y De la Torre, C. (2010). Experiencia de coordinación docente con entornos virtuales en el Grado en Administración y Dirección de Empresas en la Facultad de Ciencias Sociales de Talavera de la Reina. Revista de Estudios Económicos y Empresariales, 22, 137-147. Recuperado de: http://goo.gl/dXcvQP

Casanova, M.O, Álvarez, I.M. y Gómez, I. (2009). Propuesta de indicadores para evaluar y promover el aprendizaje cooperativo en un debate virtual. Edutec: Revista Electrónica de Tecnología Educativa, 28, 118. DOI: http://dx.doi.org/10.21556/edutec.2009.28.455

Castaño, C., Maíz, I. y Garay, U. (2015). Redes sociales y aprendizaje cooperativo en un MOOC. Revista Complutense de Educación, 26 (Núm. Especial), 119139.

DOI: http://dx.doi.org/10.5209/rev_RCED.2015.v26.463 28

Cebrián, M., Serrano, J. y Ruiz, M. (2014). Las eRúbricas en la evaluación cooperativa del aprendizaje en la Universidad. Comunicar, 43, 153-161. DOI: https://doi.org/10.3916/C43-2014-15 
Herrada Valverde, Rosario Isabel y Baños Navarro, Raúl (2018). Aprendizaje cooperativo a través de las nuevas tecnologías: Una revisión. @tic revista d'innovació educativa, 20, 16-25.

Chamizo, R. y Fernández, M.J. (2013). Nuevos métodos de intervención en el aula y en el espacio virtual: glosario y juego de rol. Historia y Comunicación Social, 18, 753-764.

DOl: http://dx.doi.org/10.5209/rev_HICS.2013.v18.44363

Corral, M.J. y Cacheiro, M.L. (2016). Los recursos TIC y el ePortfolio como estrategia para la interacción didáctica en secundaria: estudio de caso. Revista de Humanidades, 28, 115-138. Recuperado de: https://goo.gl/6DqBLC

Danker, B. (2015). Using Flipped Classroom Approach to Explore Deep Learning in Large Classrooms. IAFOR Journal of Education, 3(1), 171-186. Recuperado de: https://goo.gl/p6pJPy

De Lucas, Y., Martín, C., Haza, A.l., Delgado, C. y Lucas, J.J. (2009). RefWorks: un puente hacia la investigación en la ruta del Espacio Europeo de Enseñanza Superior. Revista Complutense de Ciencias Veterinarias, 3(1), 39-47. Recuperado de: http://goo.gl/JctEVR

Del Moral, M.E. y Villalustre. L. (2007). Ruralnet: Prácticas virtuales de aprendizaje colaborativo a través de webquest. Pixel-Bit: Revista de Medios y Educación, 29, 25-35. Recuperado de: http://goo.gl/ujLPwX

Del Pozo, M. y Horch, M. (2008). Estructuras de aprendizaje cooperativo. Cuadernos de Pedagogía, 376, 69-71. Recuperado de: http://goo.gl/6plMuc

Espinosa, I. (2014). El gato de Dalí.Escritura cooperativa con wiki. Foro de profesores de E/LE, 10, 72-82. Recuperado de: https://goo.gl/EQDnSA

Gallego, D. y Guerra, S. (2007). Las WebQuest y el aprendizaje cooperativo utilización en la docencia universitaria. Revista Complutense de Educación, 18(1), 77-94. Recuperado de: http://ir.uv.es/3R7azK7

García, A. (2008). "Edublogs" un enfoque globalizador de las competencias básicas en el currículo de lengua extranjera. Pulso: Revista de Educación, 31, 117137. Recuperado de: http://goo.gl/9elwnF

García, C. (2009). Aprendizaje cooperativo: creación de una biblioteca digital. Todo Linux: La Revista Mensual para Entusiastas de GNU/LINUX, 105, 2832. Recuperado de: http://goo.gl/m5POzN

García, A. y Suárez, C. (2011). Interacción virtual y aprendizaje cooperativo un estudio cualitativo. Revista de Educación, 354, 473-498. Recuperado de: http://goo.gl/Oq7Hix

García-Aretio, L. (2015). ¿...Y antes de los MOOC? Revista Española de Educación Comparada, 26, 2015. 97-115. DOI: 10.5944/reec.26.2015. 14775

García-Valcárcel, A. y Basilotta, V. (2017). Aprendizaje Basado en Proyectos (ABP): evaluación desde la perspectiva de alumnos de Educación Primaria. Revista de Investigación Educativa, 35(1), 113131.Recuperado de: https://goo.gl/sHw7w6

García-Valcárcel, A. y Tejedor, F.J. (2017). Percepción de los estudiantes sobre el valor de las TIC en sus estrategias de aprendizaje y su relación con el rendimiento. Educación XX1, 20 (2), 137-159. Recuperado de: http://www.redalyc.org/articulo.oa
Gómez, C., Palomares, R. y Pino, J. (2010). La utilización de herramientas colaborativas 2.0 en el ámbito de la documentación publicitaria. Revista DIM: Didáctica, Innovación y Multimedia, 18, 1-8. Recuperado de: http://goo.gl/BgMT2e

Gómez, F. (2014). La innovación educativa en la universidad a través de las TIC. ¿Qué ven los alumnos con estas prácticas? RUSC. Revista de Universidad y Sociedad del Conocimiento, 11(1), 4960. Recuperado de: http://goo.gl/bVXw2u

Gómez-Luna, Fernando-Navas, Aponte-Mayor y Betancourt-Buitrago (2014). Metodología para la revisión bibliográfica y la gestión de información de temas científicos, a través de su estructuración y sistematización. Dyna, 81(184), 158-163. Recuperado de: https://goo.gl/awVQ80

González, M.J. (2011). Innovación docente en trabajo social. El aula virtual como plataforma para el desarrollo del aprendizaje cooperativo y la investigación social. Revista de Servicios Sociales y Política Social, 96, 67-74. Recuperado de: https://goo.gl/z8cMUh

González, N. y Carrillo, G.A. (2016). El Aprendizaje Cooperativo y la Flipped Classroom: una pareja ideal mediada por las TIC. Aularia, 5(2). 43-48. Recuperado de: https://goo.gl/W24tAV

González, N., García, R. y Ramírez, A. (2015). Aprendizaje cooperativo y tutoría entre iguales en entornos virtuales universitarios. Estudios Pedagógicos, XLI(1), 111-124. Recuperado de: http://goo.gl/sXnwnZ

González, M.A., Perdomo, K.V. y Smith, O.Y. (2017). Aplicación de las TIC en modelos educativos blended learning: Una revisión sistemática de literatura, Sophia, 13(1), 144-154. DOI: https://dx.doi.org/10.18634/sophiaj.13v.1i.364

Gozálvez, V., García, R. y Traver, J.A. (2011). El aprendizaje cooperativo desde una perspectiva ética. Estudios sobre Educación, 21, 181-197. DOI/Recuperado de: http://goo.gl/uHvMHt

Guerra, S., Riaño, M.E. y González, N. (2008). La relación metodológica entre las webquest y el portafolio para la educación en valores. Pixel-Bit: Revista de Medios y Educación, 31, 179-186. Recuperado de: http://goo.gl/qeoXdJ

Guitert, M. y Pérez-Mateo, M. (2013). La colaboración en la red hacia una definición de aprendizaje colaborativo en entornos virtuales. Teoría de la Educación: Educación y Cultura en la Sociedad de la Información, 14(1), 10-31. Recuperado de: http://goo.gl/8YOhhi

Gutiérrez, P., Yuste, R., Cubo, S. y Lucero, M. (2011). Buenas prácticas en el desarrollo de trabajo colaborativo en materias TIC aplicadas a la educación. Profesorado. Revista de currículum y formación del profesorado, 15(1), 179-194. Recuperado de: http://goo.gl/PKPjv5

Hernández, M.P. (2007). Aula de español, enfoque por tareas y TIC. Algunas reflexiones sobre las webquest en la enseñanza de ELE. MarcoELE: Revista de Didáctica Español Lengua Extranjera, 5, 1-19. Recuperado de: http://goo.gl/K2670Z 
Herrada Valverde, Rosario Isabel y Baños Navarro, Raúl (2018). Aprendizaje cooperativo a través de las nuevas tecnologías: Una revisión. @tic revista d'innovació educativa, 20, 16-25.

Hernando, A., Aguaded, J.I. y Tirado, R. (2011). Aprendizaje cooperativo "on-line" a través del Campus Andaluz. Análisis de las interacciones. Enseñanza \& Teaching: Revista Interuniversitaria de Didáctica, 29(1), 135-158. Recuperado de: http://goo.gl/4XS7KX

Herrero, I., García, C., González, E., Molina, L., Pérez, E. y Urdiales, C. (2013). Aprendizaje cooperativo en el ámbito de la Ingeniería una experiencia de iniciación al Trabajo en Grupo. REDU: Revista de Docencia Universitaria, 11(Número Especial), 221-251. DOI: https://doi.org/10.4995/redu.2013.5554

Jiménez, G. y Llitjós, A.M. y Puigcerver, M. (2007). Evaluación de entornos para el aprendizaje cooperativo telemático:Synergeia. Revista Iberoamericana de Educación, 42(1), 1-16. Recuperado de: http://goo.gl/6kmxB0

Jiménez, M.A. (2016). La Rambla Aumentada: Un proyecto digital de aprendizaje-servicio. EDUTEC. Revista Electrónica de Tecnología Educativa, 58, 6576. Recuperado de: https://goo.gl/52FpUH

Johnson, D.W, Johnson, R.T. y Holubec, E.J. (1999). El aprendizaje cooperativo en el aula. Barcelona: Paidós Ibérica.

Lara, S. y Repáraz, C. (2007). Eficacia de la WebQuest para el aprendizaje cooperativo. Electronic Journal of Research in Educational Psychology, 5(13), 731-756. Recuperado de: http://goo.gl/CXHbd6

Leiva, J.J. y Almenta, E. (2013). La "digiculturalidad" o la interculturalidad a través de las TIC: Una tendencia emergente de e-learning humanizador. Etic@net: Revista Científica Electrónica de Educación y Comunicación en la Sociedad del Conocimiento, 13(1), 1-13. Recuperado de: https://goo.gl/OXAfBo

León, C., Camarillo, J., Ramos, M. y Sánchez, M.A. (2008). La enseñanza virtual en la Universidad de Sevilla. Pixel-Bit: Revista de Medios y Educación, 32, 7-20. Recuperado de: http://goo.gl/zA32cN

Leris, M. D. y Sein-Echaluce, M.L. (2009). Una experiencia de innovación docente en el ámbito universitario. Uso de las nuevas tecnologías. Arbor: Ciencia, Pensamiento y Cultura, 185, 93-110. Recuperado de: http://goo.gl/WyUQ2x

Llopis, C. (2011). Aprendizaje cooperativo. Crítica, 972, 37-41. Recuperado de: https://goo.gl/LA7wnq

López, R. y García, M.C. (2011). Libros virtuales y sus posibilidades educativas dentro de una escuela inclusiva. Revista DIM: Didáctica, Innovación y Multimedia, 20, 1-11. Recuperado de: http://goo.gl/h3J9m1

López, D. y Álvarez, I. (2011). Promover la regulación del comportamiento en tareas de aprendizaje cooperativo en línea a través de la evaluación. RIED: Revista Iberoamericana de Educación a Distancia, 14(1), 161-183. Recuperado de: http://goo.gl/kYRck0

Lozano, A. (2011). La webquest como herramienta didáctica en el desarrollo de la competencia matemática en ciencias sociales. Clío: History and History Teaching, 37, 1-111. Recuperado de: http://goo.gl/hDD42D
Maraver, P., Hernando A. y Aguaded, J.I. (2012). Análisis de las interacciones en foros de discusión a través del Campus Andaluz Virtual, @tic revista d'innovació educativa, 9, 115-123. DOI: http://dx.doi.org/10.7203/attic.9.1965

Maraza, B. (2016). Hacia un aprendizaje personalizado en ambientes virtuales, Campus Virtuales, 5(1), 2029. Recuperado de: https://goo.gl/nwu8Ra

Margelí, S. y Muria, S. (2008). Moodle, un entorno virtual colaborativo. Cuadernos de Pedagogía, 379, 60-61. Recuperado de: http://goo.gl/vpHyTG

Mateo, F. (2015). Producción científica en español en humanidades y ciencias sociales. Algunas propuestas desde Dialnet. El Profesional de la Información, 24(5), 509-515. Recuperado de: https://goo.gl/9NDcNy

Méndez, D. (2015). Estudio de las motivaciones de los estudiantes de secundaria de física y química y la influencia de las metodologías de enseñanza en su interés. Educación XX1, 18(2), 215-235. DOI: http://dx.doi.org/10.5944/educXX1.14016

Miguel, F.J. (2010). Una experiencia de comunidad de aprendizaje virtual. El uso de nuevas TIC en el desarrollo colaborativo de recursos WWW para una asignatura. Papers: Revista de Sociología, 95(4), 1175-1186. Recuperado de: http://goo.gl/rrlzF5

Molina, P., Valencia-Peris, A. y Gómez-Gonzalvo, F. (2016). Innovación docente en educación superior: Edublogs, evaluación formativa y aprendizaje colaborativo. Profesorado: Revista de Curriculum y Formación del Profesorado, 20(2), 432-450. Recuperado de: https://goo.gl/KaP7z1

Mondéjar, J., Vargas, M. y Meseguer, M.L. (2007). Aprendizaje Cooperativo en Entornos Virtuales el Método Jigsaw en Asignaturas de Estadística. Documentos de Trabajo.Seminario Permanente de Ciencias Sociales, 3, 1-18. Recuperado de: http://goo.gl/i4RmZh

Monedero, C., Castro, A. y Luengo, J.T. (2017). Videotutoriales y códigos QR: recursos TIC en laboratorios de Ciencias de la Comunicación. Innoeduca: International Journal of Technology and Educational Innovation, 3(2), 137-145. DOI: http://dx.doi.org/10.24310/innoeduca.2017.v3i2.2 046

Moreno-Crespo, P., López, F. y Cruz, M.R. (2014). Portafolio digital: un nuevo formato de aprendizaje. Didáctica de las Ciencias Experimentales y Sociales, 28, 83-94. Recuperado de: https://goo.gl/ImpOIA

Niculcar, C.A.E., Fernández, J., Gracia, A., García, A. y González Benítez, M. (2009). Metodología de diseño de proyectos de ingeniería química a partir del fomento del aprendizaje cooperativo. Afinidad: Revista de Química Teórica y Aplicada, 668(539), 714. Recuperado de: http://goo.gl/latGLZ

Nuñez, T.F. (2011). Entornos virtuales de enseñanza aprendizaje (EVEA): Formación profesional. Edutec-e, Revista Electrónica de Tecnología Educativa, 37, 115. DOI: http://dx.doi.org/10.21556/edutec.2011.37.389 
Herrada Valverde, Rosario Isabel y Baños Navarro, Raúl (2018). Aprendizaje cooperativo a través de las nuevas tecnologías: Una revisión. @tic revista d'innovació educativa, 20, 16-25.

Ortega, A. (2015). Valoración de actividades de aprendizaje cooperativo formal bilingüe en el ámbito universitario. IJERI: International Journal of Educational Research and Innovation, 4, 12-25. Recuperado de: http://goo.gl/smwPjp

Osicka, R.M., Fernández, M.L., Valenzuela. A.M., Buchhamer, E.E. y Giménez, M.C. (2013). Química analítica: aprendizaje a partir de WebQuest. Avances en Ciencias e Ingeniería, 4(1), 131-138. Recuperado de: https://goo.gl/sDfhCv

Palomares, D. y Chisvert, M.J. (2016). El aprendizaje cooperativo: una innovación metodológica en la formación del profesorado Cultura y Educación: Culture and Education, 28(2), 387-395

Pantoja, A. y Blanco, E. (2011). Aprendizaje cooperativo y heurístico en la red internacional e-Culturas. Teoría de la Educación: Educación y Cultura en la Sociedad de la Información, 12(4), 39-52. Recuperado de: http://goo.gl/AcNS8N

Pegalajar, M.C. (2009). Las nuevas tecnologías de la información y la comunicación en el aprendizaje de alumnos con discapacidad intelectual. Portularia: Revista de Trabajo Social, 9(Número Extraordinario), 47-51. Recuperado de: http://goo.gl/SpKaD9

Peña, K., Pérez, M. y Rondón. E. (2010). Redes sociales en Internet: reflexiones sobre sus posibilidades para el aprendizaje cooperativo y colaborativo. Revista de Teoría y Didáctica de las Ciencias Sociales, 16, 173205. Recuperado de: http://goo.gl/64vYIM

Pujolàs, P. (2008). El aprendizaje cooperativo como recurso y como contenido. Aula de Innovación Educativa, 170, 37-41. Recuperado de: https://goo.gl/pFa4sQ

Pulido, R., Martín-Seoane, G. y Lucas-Molina, B. (2013). Orígenes de los programas de mediación escolar: Distintos enfoques que influyen en esta práctica restaurativa. Anales de Psicología, 29(2), 385-392. DOI: http://dx.doi.org/10.6018/analesps.29.2.132601

Rodríguez, J.C. y De Miguel, T.P. (2007). Titulaciones conjuntas en ambientes cooperativos de e-learning. RUSC. Revista de Universidad y Sociedad del Conocimiento, 4(2), 1-11. Recuperado de: http://goo.gl/OrCMlu

Rodríguez, L.M., Cebollada, J.L., Hueto, M.A. y Molledo, J. (2012). Aprendizaje cooperativo y TIC. Comunicación y Pedagogía: Nuevas Tecnologías y Recursos Didácticos, 261-262, 51-53. Recuperado de: https://goo.gl/eFsaSX

Ros, I. (2008). Moodle, la plataforma para la enseñanza y organización escolar. Ikastorratza, e-Revista de Didáctica, 2, 1-12. Recuperado de: http://goo.gl/oxs0t4

Roselli, N.D., Bruno, M. y Evangelista, L. (2004). El chateo y la interacción social directa en el aprendizaje cooperativo de díadas. Revista Latinoamericana de Psicología, 36(3), 391-408. Recuperado de: http://goo.gl/1JsYqF

Ruda, A. (2013). Percepción de los estudiantes sobre el uso de wiki. Análisis de una experiencia de aprendizaje colaborativo en la Universitat de Girona.
Teoría de la Educación: Educación y Cultura en la Sociedad de la Información, 14(1), 76-100. Recuperado de: http://goo.gl/ny9cLS

Sáez, J.M. (2010). Utilización de las TIC en el proceso de enseñanza aprendizaje, valorando la incidencia real de las tecnologías en la práctica docente. Docencia e Investigación, 10, 183-204. Recuperado de: http://goo.gl/XOqxrv

Salmerón, H., Rodríguez, S. y Gutiérrez, C. (2010). Metodologías que optimizan la comunicación en entornos de aprendizaje virtual. Comunicar, 34(17), 163-171. Recuperado de: http://goo.gl/xYn5I5

Sánchez-Vera, M.M., González, V. y Prendes, M.P. (2017). Los MOOC y la evaluación del alumnado: revisión sistemática (2012-2016). @tic revista d'innovació educativa, $18, \quad 65-73 . \quad$ DOI: http://doi.org/10.7203/attic.18.10013

Sánchez-Vera, M.M, Solano, I.M. y González, V. (2016). FLIPPED-TIC: Una experiencia de Flipped Classroom con alumnos de Magisterio. RELATEC: Revista Latinoamericana de Tecnología Educativa, 15(3), 5567.

DOI: http://doi.org/10.17398/1695-288X.15.3.69

Sánchez, M.L. (2014). Diseño y producción de cursos MOOC como estrategia de aprendizaje cooperativo en un ambiente de educación a distancia. Revista DIM: Didáctica, Innovación y Multimedia, 28, 1-12. Recuperado de: http://goo.gl/zbPnOj

Santillán-Campos, F. (2013). El Aprendizaje Basado en Problemas como propuesta educativa para las disciplinas económicas y sociales apoyadas en el BLearning Revista Iberoamericana de Educación, 40(2). Recuperado de: https://goo.gl/LDtq2N

Sarabia, C.M. (2016). Nuevas culturas educativas.Los MOOCs en las universidades españolas. Cultura y Educación, 28(1), 204-212.

Serrano, R., Muñoz, J.M. y López, I. (2012). Valoración por los estudiantes de la Licenciatura en Psicopedagogía de una experiencia innovadora basada en estrategias de aprendizaje colaborativo y el uso del Courselab. Teoría de la Educación: Educación y Cultura en la Sociedad de la Información, 13(1), 437-458. Recuperado de: http://goo.gl/OkZOmR

Siliberti, A. (2011). Propuesta de un modelo de innovación docente aplicado a la enseñanza del italiano como lengua extranjera. Hekademos: Revista Educativa Digital, 8, 91-102. Recuperado de: https://goo.gl/4gJPJs

Silva, J. (2017). Un modelo pedagógico virtual centrado en las E-actividades. RED. Revista de Educación a Distancia, 53, artículo 10, 1-20. DOI: http://dx.doi.org/10.6018/red/53/10

Suárez, C. (2010). Aprendizaje cooperativo e interacción asíncrona textual en contextos educativos virtuales. Pixel-Bit: Revista de Medios y Educación, 36, 53-67. Recuperado de: http://goo.gl/3Y51eh

Tirado, R., Aguaded, J.I., y Méndez J.M. (2009). Interacciones en grupos de aprendizaje on line. Revista Iberoamericana de Educación, 48(5), 1-17. Recuperado de: http://goo.gl/NBHr7y 
Herrada Valverde, Rosario Isabel y Baños Navarro, Raúl (2018). Aprendizaje cooperativo a través de las nuevas tecnologías: Una revisión. @tic revista d'innovació educativa, 20, 16-25.

Tirado, R., Hernando, A. y Aguaded, J.I. (2011). Aprendizaje cooperativo on-line a través de foros en un contexto universitario: un análisis del discurso y de las redes. Estudios sobre Educación, 20, 49-71. Recuperado de: http://goo.gl/PoJMC2

Trigueros, C., Rivera, E. y De la Torre, E. (2011). El Chat como estrategia para fomentar el aprendizaje cooperativo. Una investigación en el Prácticum de Magisterio. Profesorado: Revista de Curriculum y Formación del Profesorado, 15(1), 195-210. Recuperado de: http://goo.gl/bvEkhr

Trujillo, J.M., Cáceres, M.P., Hinojo, F.J. y Aznar, I. (2011). Aprendizaje cooperativo en entornos virtuales. El proyecto Redes Educativas y Organizativas Interuniversitarias. Educar, 47(1), 95-119. Recuperado de: http://goo.gl/wmxBY2

Zanfrillo, A.I. y Díaz, M.D. (2015). En coordenadas de virtualidad: una experiencia interdisciplinaria entre instituciones de educación superior. FACES: Revista de la Facultad de Ciencias Económicas y Sociales, 21(44), 69-86. Recuperado de: http://goo.gl/uxx70q

| Cita recomendada de este artículo

Herrada Valverde, Rosario Isabel y Baños Navarro, Raúl (2018). Aprendizaje cooperativo a través de las nuevas tecnologías: Una revisión. @tic revista d'innovació educativa, 20, 16-25. 